\title{
BF STEM Tomography for Improved 3D Imaging of Thick Biological Sections
}

\author{
A.A. Sousa*, M.F. Hohmann-Marriott**, A. Azari*, G. Zhang*, R.D. Leapman* \\ *Laboratory of Bioengineering and Physical Science, NIBIB, NIH, Bethesda MD \\ **University of Otago, Department of Biochemistry, Dunedin, New Zealand
}

Electron tomography (ET) of stained biological specimens is typically performed from a series of bright-field (BF) images recorded over a range of tilt angles in a transmission electron microscope. In order to obtain an adequate resolution in electron tomograms that allow visualization of nanoscale macromolecular organization, conventional ET is typically constrained to relatively thin sections of around $200 \mathrm{~nm}$ [1]. ET based on scanning transmission electron microscopy (STEM) is an attractive approach with which to obtain 3D reconstructions from thick sections at higher resolutions than are possible with conventional ET. In STEM there are no image-forming lenses after the specimen, so resolution is not degraded by chromatic aberration. Here, we demonstrate that with BF STEM it is possible to generate tomograms from $1 \mu \mathrm{m}$-thick biological specimens at a resolution of $10 \mathrm{~nm}$.

STEM tomography of micron-thick specimens is optimally performed under a rather different electron-optical configuration than the one typically associated with high-resolution STEM imaging of thin specimens. In particular, STEM of thin specimens gives rise to optimum resolution by using large convergence angles of the incident probe (10-40 mrad), and optimum contrast by using large collection angles of the HAADF detector (inner semi-angle $\geq 40 \mathrm{mrad}$ ) [2]. For imaging micronthick sections, however, the convergence angle needs to be adjusted to 1-2 mrad to maximize depth of field [3]. Moreover, the use of a BF detector of small outer semi-angle ( $15 \mathrm{mrad})$ gives rise to improved resolution at the bottom regions (towards the beam exit surface) [4].

Fig. 1a shows calculated values of beam diameter at the bottom of specimens of various thicknesses. The calculations were done with Monte Carlo simulations [5] and have been described elsewhere [4]. As expected, the beam diameter increases significantly with thickness due to multiple elastic scattering. Fig. 1a reveals however that this degradation in spatial resolution is much less significant in BF STEM, which is due to a large fraction of the multiply scattered electrons being excluded from the axial BF detector [4]. Images of gold particles at the top and bottom surfaces of a thick section were recorded with both BF and HAADF STEM (Fig. 1b). The particles situated at the top of the section (beam entrance surface) appear the same in both imaging modes, whereas the ones situated at the bottom (arrows in Fig. 1b) are visualized at much higher resolution with BF STEM.

We have evaluated the improved STEM tomography approach, which uses an incident probe of low convergence angle and a BF detector for data acquisition, in reconstructions of a $1 \mu \mathrm{m}$-thick section of Chlamydomonas reinhardtii. This organism contains stacked photosynthetic membranes making it an ideal object to assess resolution in a tomogram. First, we recorded a BF STEM tomogram that encompassed an entire $C$. reinhardtii cell in the $x y$ plane (Fig. 2a). Next, we obtained a higher resolution tomogram from a selected $4 \mu \mathrm{m}^{2}$ area of the cell (Fig. 2b). Stacked membranes separated by $\sim 10 \mathrm{~nm}$ are easily visible, revealing that resolution in the tomogram is better than $10 \mathrm{~nm}$. The ability to perform 3D reconstructions from larger volumes and at higher resolutions than are achievable with existing methods could facilitate the study of entire unicellular eukaryotic microorganisms that can be contained within only 1 to 4 serial $1 \mu \mathrm{m}$-thick sections. 
[1] R. McIntosh et al., Trends Cell Biol. 15 (2005) 43.

[2] P.E. Batson et al., Nature 418 (2002) 617.

[3] J.K. Hyun et al., Ultramicroscopy 109 (2008) 1.

[4] A.A. Sousa et al., Ultramicroscopy (2009) In press.

[5] D.C. Joy, Monte Carlo Modeling, Oxford Univ. Press, NY, 1995.

[6] Research supported by the intramural program of NIBIB, NIH
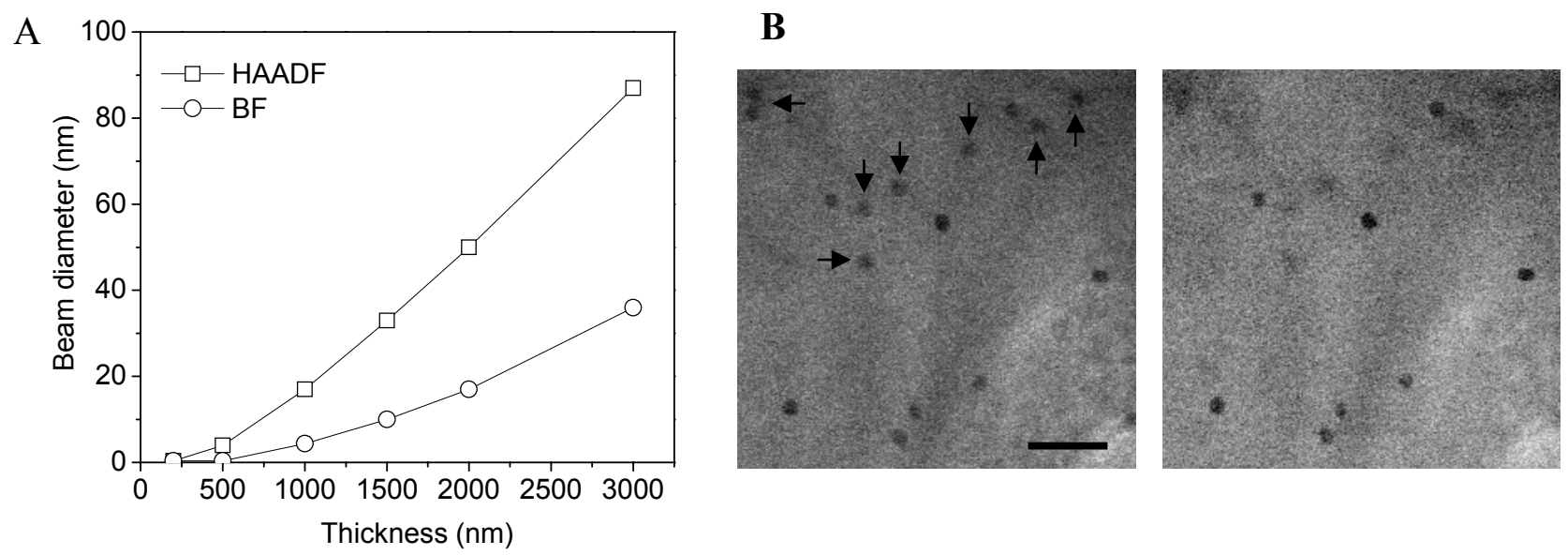

Fig. 1. (A) Diameter of electron probe (at 50\% integrated intensity) at the bottom of specimens of various thicknesses calculated using Monte Carlo simulations [4]. BF detector: $10 \mathrm{mrad}$ outer semiangle. HAADF: 40 mrad inner semi-angle. (B) BF (left) and HAADF (right) projection images of gold particles situated at the top and bottom of a densely stained $1 \mu \mathrm{m}$-thick plastic section. HAADF is displayed with inverted contrast. As predicted by calculations, BF STEM leads to a significantly better resolution of the gold particles located at the bottom (arrows). Scale $=100 \mathrm{~nm}$.
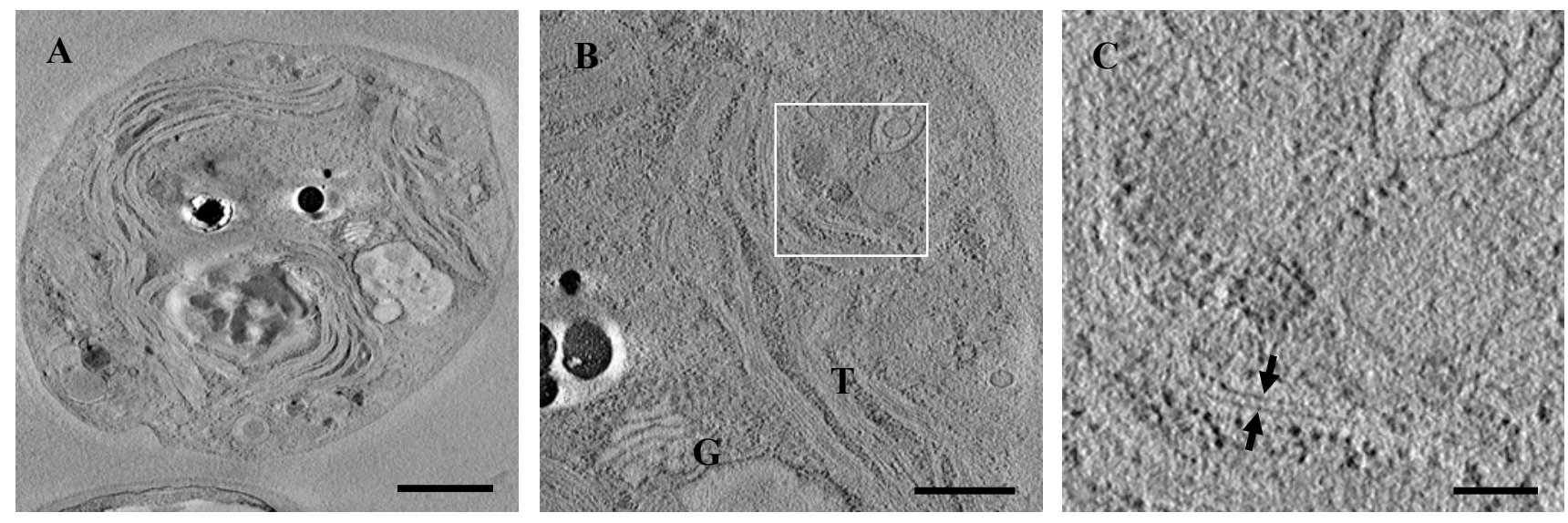

Fig. 2. (A) Slice across a reconstructed STEM tomogram obtained from a $1 \mu \mathrm{m}$-thick plastic embedded and stained section of $C$. reinhardtii. Data were acquired with an incident probe of 1.6 mrad convergence semi-angle and a $\mathrm{BF}$ detector of $\sim 15 \mathrm{mrad}$ outer semi-angle. Pixel size is $5.7 \mathrm{~nm}$ after binning. (B) Slice across a tomogram recorded from a $4 \mu \mathrm{m}^{2}$ selected region of the cell. Pixel size is $2 \mathrm{~nm}$ after binning. Thylakoid stacks (T), a Golgi apparatus (G), and several vesicles are well visible. (C) Expanded area from (b): two membranes (arrows) separated by 10-12 nm are visible with high contrast in the tomogram, indicating that the resolution of the dataset is better than $10 \mathrm{~nm}$. Scale bars are $1 \mu \mathrm{m}$ in (A), $400 \mathrm{~nm}$ in (B) and $100 \mathrm{~nm}$ in (C). 